ARTICLE

Received 20 Sep 2013 | Accepted 7 May 2014 | Published 6 Jun 2014

DOI: $10.1038 /$ ncomms5054

OPEN

\title{
Dynamic haematopoietic cell contribution to the developing and adult epicardium
}

\author{
Gemma M. Balmer ${ }^{1, \star}$, Sveva Bollini ${ }^{2, \star}$, Karina N. Dubé ${ }^{1}$ Juan Pedro Martinez-Barbera ${ }^{3}$, Owen Williams ${ }^{4}$ \\ $\&$ Paul R. Riley 5
}

The epicardium is a cellular source with the potential to reconstitute lost cardiovascular tissue following myocardial infarction. Here we show that the adult epicardium contains a population of CD45 + haematopoietic cells (HCs), which are located proximal to coronary vessels and encased by extracellular matrix (ECM). This complex tertiary structure is established during the regenerative window between post-natal days 1 and 7 . We show that these $\mathrm{HCs}$ proliferate within the first $24 \mathrm{~h}$ and are released between days 2 and 7 after myocardial infarction. The ECM subsequently reforms to encapsulate HCs after 21 days. Vav1-tdTomato labelling reveals an integral contribution of CD45 + HCs to the developing epicardium, which is not derived from the proepicardial organ. Transplantation experiments with either whole bone marrow or a Vav1 + subpopulation of cells confirm a contribution of HCs to the intact adult epicardium, which is elevated during the first 24 weeks of adult life but depleted in aged mice.

\footnotetext{
${ }^{1}$ Molecular Medicine Unit, UCL-Institute of Child Health, London WC1N 1EH, UK. ${ }^{2}$ Regenerative Medicine Laboratory, University of Genoa \& IRCCS AOU San Martino-IST, Largo Rosanna Benzi, 16132 Genoa, Italy. ${ }^{3}$ Neural Development Unit, UCL-Institute of Child Health, London WC1N 1EH, UK. ${ }^{4}$ Molecular Haematology and Cancer Biology Unit, UCL-Institute of Child Health, London WC1N 1EH, UK. ${ }^{5}$ Department of Physiology, Anatomy and Genetics, University of Oxford, Oxford OX1 3PT, UK. * These authors contributed equally to this work. Correspondence and requests for materials should be addressed to P.R.R. (email: paul.riley@dpag.ox.ac.uk).
} 
$\mathrm{n}$ the adult mammalian heart, the existence of resident stem cells, which can act to restore lost tissue after a heart attack (myocardial infarction $(\mathrm{MI}))^{1}$, is the subject of ongoing investigation. The adult epicardium, classically described as a single cell layer epithelium (mesothelium) lining the chamber myocardium of the heart, has emerged in recent times as a putative resident source of cardiovascular progenitors, which when appropriately activated, for example, by pharmacological treatment with factors such as thymosin $\beta 4$, can contribute to neovascularisation and myocardial regeneration of the injured adult mouse heart ${ }^{2,3}$. In addition, the adult epicardium also acts as an important source of trophic signals and has been shown to secrete a number of key angiogenic signals to instruct new coronary vessel growth following $\mathrm{MI}^{4}$. The application of epicardial cell biology to the treatment of cardiovascular injury originates from the epicardium's developmental potential and from the ability to reactivate these properties in the adult heart. During heart development, the epicardium arises from a transient structure called the proepicardial organ (PEO), of mesoderm origin, located at the inflow region of the developing heart tube above the primordial liver (reviewed in ref. 5). As the heart begins to loop (E8.5-9.0 in mouse), PEO cells undergo epithelialmesenchymal transition to migrate towards the developing myocardium and envelop it to form the epicardium proper. This is then followed by a successive round of epithelialmesenchymal transition, whereby epicardium-derived cells (EPDCs) migrate into the sub-epicardial region and contribute interstitial fibroblasts, vascular smooth muscle cells and, to a lesser extent, coronary endothelial cells and cardiomyocytes to the embryonic heart. The retained fetal epicardial layer also provides growth factors and cytokines to nurture the growth of the underlying myocardium (reviewed in ref. 6). A re-expression of embryonic epicardial genes (characterized by the signature genes $W t 1, T b \times 18$ and Raldh2) initiates adult EPDC activation ${ }^{3,4}$ and is a hallmark of the downstream contribution of EPDCs in response to MI. The plasticity of EPDCs in the injury setting ${ }^{2-4,7}$ prompted us to investigate whether the adult epicardium might be a more complex structure than the previously described simple mesothelium, and whether it might house heterogeneous cell types to act as a regenerative reserve during cardiovascular homeostasis and in response to stress or pathology.

Thus far, the potential of the adult epicardium to be reactivated and contribute to cardiac repair has been realized entirely in the context of reactivation of a dormant, homogeneous single epithelial cell layer. Our findings suggest the epicardium is not a simple mesothelium but is, in fact, made up of discrete clusters of heterogeneous cell types, which include a novel haematopoietic contribution of distinct origin, encased by extracellular matrix (ECM) that anatomically resembles a stem or progenitor cell 'niche'. This establishes the basis for a re-evaluation of the reparative potential of the adult epicardial lineage. Moreover, a haematopoietic cell (HC) contribution to the epicardium during development and adult homeostasis contrasts with the canonical view of a single $\mathrm{PEO}$ source maintained in steady state throughout adulthood. Consequently, the complex architecture of the adult epicardium and turnover of diverse constituent cell types offers the possibility of pharmacological targeting in cardiovascular repair.

\section{Results}

Adult epicardial cell clusters encased by extracellular matrix. We first analysed intact hearts and, contrary to the prevailing view of the epicardium as a single cell layer, observed small cells with large nuclei-cytoplasmic ratios in situ, at intervals throughout the ventricular chambers and contiguous with the epicardium, as defined by the surface glycoprotein podoplanin ${ }^{3}$ (Fig. 1a-c). These cell clusters were held in suspension via basement membrane proteins and key components of the ECM such as fibronectin (Fig. 1d,e; Supplementary Fig. 1a-f), $\beta 1$-integrin (CD29; Fig. 1f), collagen IV (Supplementary Movie 1 of a confocal $Z$-stack projection) and hyaluronic acid (Supplementary Fig. 1g-r). The cells within these apparent 'microenvironments' contained a population defined by specific expression of the cell surface glycoprotein CD44 (ref. 8), previously shown to mark a population of $\mathrm{PEO}$ origin that was proposed to give rise to mesenchymal-like stem cells residing within the adult epicardium 9 (Fig. 1g). Further characterization revealed the presence of distinct CD45 + HCs (Fig. 1h), which represented the more predominant resident population. Epicardium cell clusters were localized in close proximity to superficial coronary vessels (Fig. 1i,j), including those that contained CD45 + HCs (Fig. 1k). Each cluster consisted of between 3 and 15 CD $45+$ cells; the mean number of cells per cluster was $6.6 \pm 1.8$ s.e.m. $(n=25$ sections across 5 hearts, at 8 weeks of age) and there were $2.12 \pm 0.26$ clusters per $\mathrm{mm}$ of epicardium (mean circumference of epicardium/total number of cell clusters/section \pm s.e.m.; $n=25$ sections analysed across 5 hearts, at 8 weeks of age; $P<0.01$; Student's $t$-test). These data suggest the epicardium is not a simple mesothelium ${ }^{10}$, but contains structures that represent a heterogeneous mix of cell populations, including HCs, with vascular support and a non-cellular contribution of ECM.

Epicardial clusters form during early neonatal stages. The three-dimensional geometry of the clusters (Supplementary Movie 1) was indicative of tertiary structure within the adult epicardium, over and above the primary cell constituents and secondary two-dimensional organization of the surface epithelium. To determine when this tertiary structure might be established, we focused on early post-natal stages (P) and specifically between days 1 to 7 after birth. This represents a critical window of time on two counts: first, the potential of the epicardium during development is lost at around P4 in terms of an inherent ability of EPDCs to migrate, contribute cardiovascular derivatives and trophic activity ${ }^{11}$, and second, the first week post birth is consistent with loss of regenerative capacity of the heart during P1 to P7. Myocardium was regenerated following ventricular resection injury at P1, but fibrosis and scarring ensued at P7, and although this switch was accounted for by a loss of proliferative potential of resident cardiomyocytes during this time frame, a contribution from a stem/progenitor cell component could not be excluded ${ }^{12}$. At P1, ECM components, such as fibronectin, were expressed widely throughout the heart and there were no apparent distinct clusters of cells within the epicardium (Fig. 2a), rather a dispersed incidence of CD44 + and CD45+ cells, which lacked any anatomical constraint (Fig. 2b,c). In contrast, by P7 we observed evidence of nascent epicardial ECM, encapsulating small numbers of $\mathrm{CD} 44+$ and $\mathrm{CD} 45+$ cells (Fig. 2d-f). There was no evidence of increased CD44 + or CD45 + cells during this post-natal window, but simply a reorganization into discrete ECM-defined clusters. Thus, the relatively complex epicardial topology appeared to be initiated during the first week after birth, putatively reflecting a more limited stem cell potential, which may in turn contribute to the transient regenerative capacity of the neonatal mouse heart.

A dynamic $\mathrm{CD} 45+$ cell and ECM response to ischaemic injury. We next determined whether the adult epicardium modulated cell function through structural remodelling under conditions of physiological challenge or pathology ${ }^{13}$. Our focus 

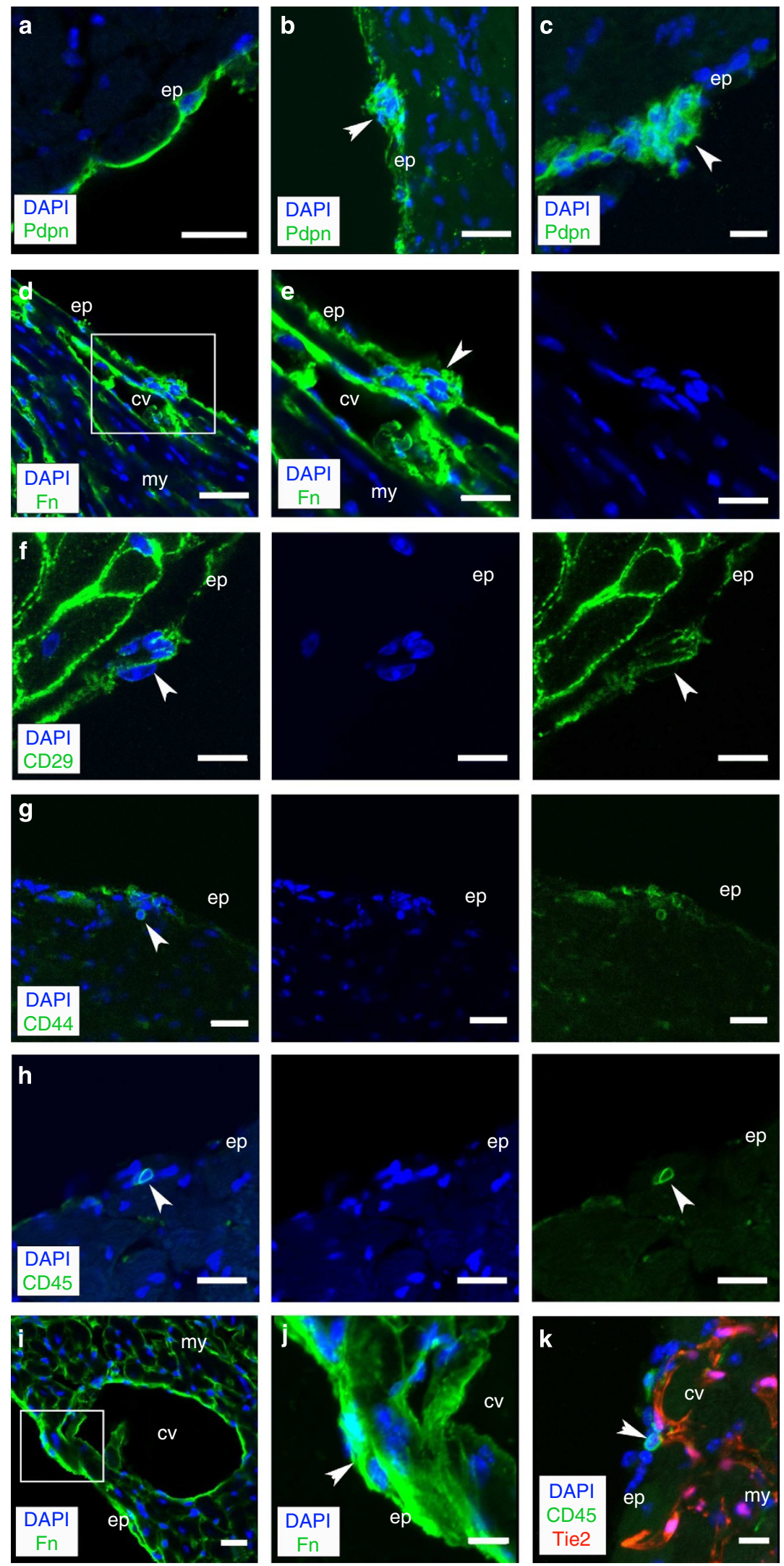

Figure 1 | Clusters of cells constrained by ECM exist in the intact adult epicardium. Immunofluorescent staining (IMF) for podoplanin (Pdpn; green) in the intact adult epicardium identifies clusters of cells within basement membrane (white arrowheads; a-c). Clusters in the epicardium (white arrowheads) are encapsulated by ECM as indicated by IMF for fibronectin (Fn; green; $\mathbf{d}$ ). Inset box in $\mathbf{d}$ is shown at higher magnification in $\mathbf{e}$, and CD29 ( $\beta 1$-integrin; f). IMF for CD44 and CD45 revealed the clusters were a heterogeneous population of mesenchymal (CD44; $\mathbf{g})$ and HCs (CD45; $\mathbf{h})$. The clusters resided in close proximity to coronary vessels $(\mathbf{i}, \mathbf{j})$ as confirmed by immunostaining on a Tie2Cre;R26R-tdTomato background (k). $\mathrm{cv}$, coronary vessel; ep, epicardium; my, myocardium. Scale bars, $40 \mu \mathrm{m}$ (a); $20 \mu \mathrm{m}(\mathbf{b}-\mathbf{e}) ; 10 \mu \mathrm{m}$ (f-k). 
here was on the novel $\mathrm{CD} 45+$ population given the previous description of epicardium-derived CD44-derived mesenchymal stem cell-like cells ${ }^{9}$. MI can cause extensive epicardial fibrosis ${ }^{14}$ and, consequently, analyses were carried out on neighbouring intact epicardium, as previously investigated ${ }^{3,4,15}$. After MI, we observed that resident CD $45+$ cells began to proliferate at $24 \mathrm{~h}$ as detected by pulse chase (injections at 4,8 and $22 \mathrm{~h}$ post $\mathrm{MI}$ ) with 5-bromo-2'-deoxyuridine (BrdU; Fig. 3a,b) and co-staining with Ki67 (Fig. 3c-f). The numbers of proliferating cells per cluster ranged from 2 to 5 and the mean number of $\mathrm{BrdU}+$ or Ki67 + cells was $3.2 \pm 0.6$ s.e.m. $(n=15$ sections, across 6 hearts at 8 weeks of age). Subsequently, the ECM boundary appeared to break down, within the first 2 days, as best illustrated by a downregulation of fibronectin protein expression leading to loss of epicardial integrity (Fig. 3g). ECM breakdown was supported by co-localization of matrix metalloproteinase 2 and 9 expression at corresponding stages post MI (Supplementary Fig. 2a-d). This breakdown persisted to day 7 post MI and was accompanied by concomitant expansion of CD45 + cells (Fig. 3i,k;) and their release into the subepicardial region and underlying myocardium (Fig. 3k, right hand panel). Interestingly, regional differences in epicardial response were observed, with disassembly of ECM structures proximal to the site of injury in the left ventricle (LV), whereas those more distal in the right ventricle remained relatively intact, even up to 7 days after injury (Fig. 3h,j,l), indicating a spatial gradient in response to injury signalling. After 21 days post $\mathrm{MI}$, the tertiary structure reformed in the LV with restoration of CD $44+$ cells (Fig. $3 \mathrm{~m}$ ) and CD $45+$ cells (Fig. 3n), such that by day 42 there was a return to the pre-injury quiescent state (compare Figs $1 \mathrm{a}-\mathrm{c}$ and $3 \mathrm{o}$ ) and comparable to that observed throughout in the right ventricle (Fig. 3p). Collectively, these data suggest that the adult epicardium is a dynamic structure, which responds to injury signals from the infarcted LV to break down the local ECM and release proliferative progenitors into the underlying injured myocardium.

Canonical Wt1 + epicardial cells are distinct from HC clusters. We next investigated the relative spatial localization of reactivated $\mathrm{Wt} 1+$ cells, previously identified as an important subpopulation of epicardial progenitors following $\mathrm{MI}^{3,4}$, within the higher-order structure of the adult epicardium. Injury-activated Wt $1+$ cells were distinct from the resident $\mathrm{CD} 45+\mathrm{HC}$ population (Fig. 4a,b) and, although they were not localized within the clusters they resided immediately proximal (Fig. 4c,d). The response of the $\mathrm{Wt} 1+$ population varied considerably depending on the extent of infarction and injury, as previously reported ${ }^{3,4}$. Following injury (day 4 post MI), the breakdown of the ECM clusters and expansion of the sub-epicardial region contained some Wt1 + cells, as reported by Wt1CreERT2;R26R-tdTomato lineage tracing from developmental stages alongside immunostaining for $\mathrm{Wt1}$ (Fig. 4e,f). Efficient recombination of the R26R-tdTomato, by tamoxifen-induced Wt1CreERT2, was observed with specific tdTomato + reporter labelling of the epicardium throughout development and into adulthood (Supplementary Fig. 3a-l), as well as efficient tracing of $\mathrm{Wt} 1+$ cell derivatives, such as smooth muscle cells, within the adult heart (Supplementary Fig. 4a-d). Wt1-tdTomato + cells were also evident throughout the myocardium (Fig. 4e), reflecting the increased contribution of $\mathrm{Wt1}$ derivatives over time (Supplementary Fig. 3h-l). The Wt1-negative cells in the
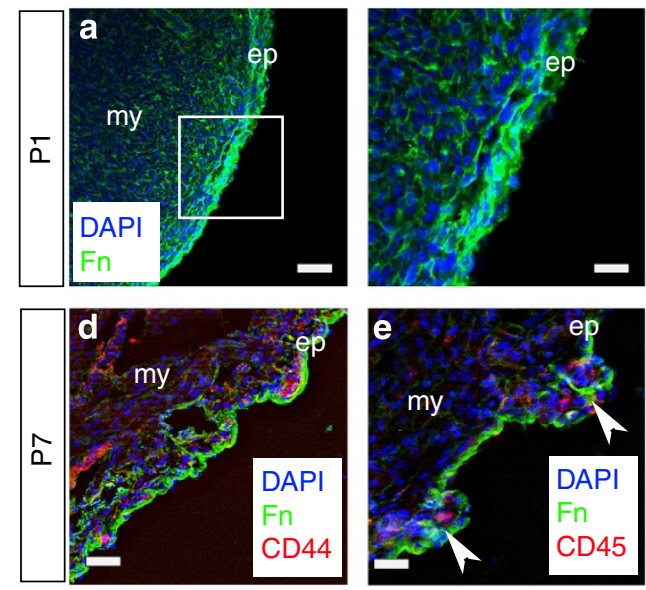
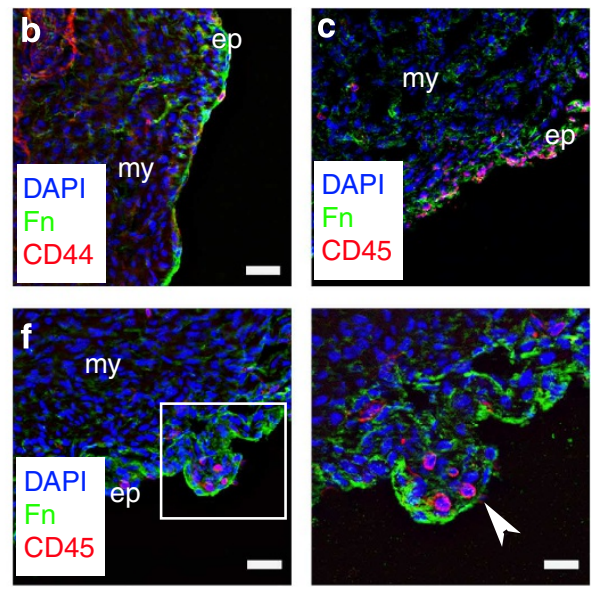

Figure 2 | Tertiary epicardial structure forms during early post-natal stages. At post-natal day 1 (P1), there is no evidence of cell clusters or higher-order structure in the epicardium; Fn staining is diffused throughout (a), and the CD44+ and CD45+ cells that are present do not occupy discrete locations within the epicardium $(\mathbf{b}, \mathbf{c})$. By P7, there is evidence of Fn surrounding the forming clusters containing CD44 $+(\mathbf{d})$ and CD45 + cells (e,f; clusters highlighted by white arrowheads). White inset boxes in the left panels are highlighted at higher magnification in neighbouring right panels. ep, epicardium; my, myocardium. Scale bars,: $50 \mu \mathrm{m}$ (a, left panel); $20 \mu \mathrm{m}$ (a, right panel); $30 \mu \mathrm{m}$ (b-f, left panel); $20 \mu \mathrm{m}$ (f, right panel).

Figure 3 | Epicardial HC clusters respond dynamically to myocardial injury. Following Ml, there is an initial proliferative response as determined by BrdU + pulse labelling of cluster cells 1 day post injury (1 d.p.i.) in both the left (LV) and right (RV) ventricles (white arrowheads highlight BrdU + cells; a,b). CD 45 + cells located within the epicardial layer were confirmed as undergoing cell cycle activity via Ki67 co-staining in both the LV (c,d) and RV (e,f; white arrowheads highlight CD45 + /Ki67 + cells). As early as day 2 post MI (2 d.p.i.) in the LV, the clusters begin to break down (g). Disassembly of ECM-encapsulated cell clusters persists through day 4 (4 d.p.i.; i) and progresses to day 7 (7 d.p.i.; $\mathbf{k}$ ) to release CD45 + cells ( $\mathbf{g}, \mathbf{i}, \mathbf{k}$ ) This is not the case in the RV where the clusters remain intact over the same time course of injury constraining the resident cell types (h,j, I); white arrowhead in I highlights intact cluster 7 d.p.i. By day 21 post MI (21 d.p.i.), the clusters have reformed (white arrowheads in higher magnification view highlight Fn at the apical surface) in the LV (m) to resemble the persistent intact clusters of the RV (n) and pre-injury state (Fig. 1); the reinstated 'niche' is evident 42 days post MI (42 d.p.i.; $\mathbf{0}$ ) and is comparable to that in the RV as highlighted by white arrowheads (p). White inset boxes in the left panels are highlighted at higher magnification in neighbouring right panels. ep, epicardium; my, myocardium. Scale bars, $50 \mu \mathrm{m}$ (a-p, left panels); $20 \mu \mathrm{m}$ (g,i,j,l, $\mathbf{n}) ; 10 \mu \mathrm{m}$ (a,b,h,k, m-p, right panels). 
sub-epicardial region were predominantly CD45 + (Fig. 4g,h), suggesting an influx of $\mathrm{HCs}$ consistent with an early pro-inflammatory response ${ }^{16}$. The extent of $\mathrm{CD} 45+$ cellular contribution to the expanded epicardium at day 4 post MI was comparable to that observed within the bone marrow (Supplementary Fig. 5).
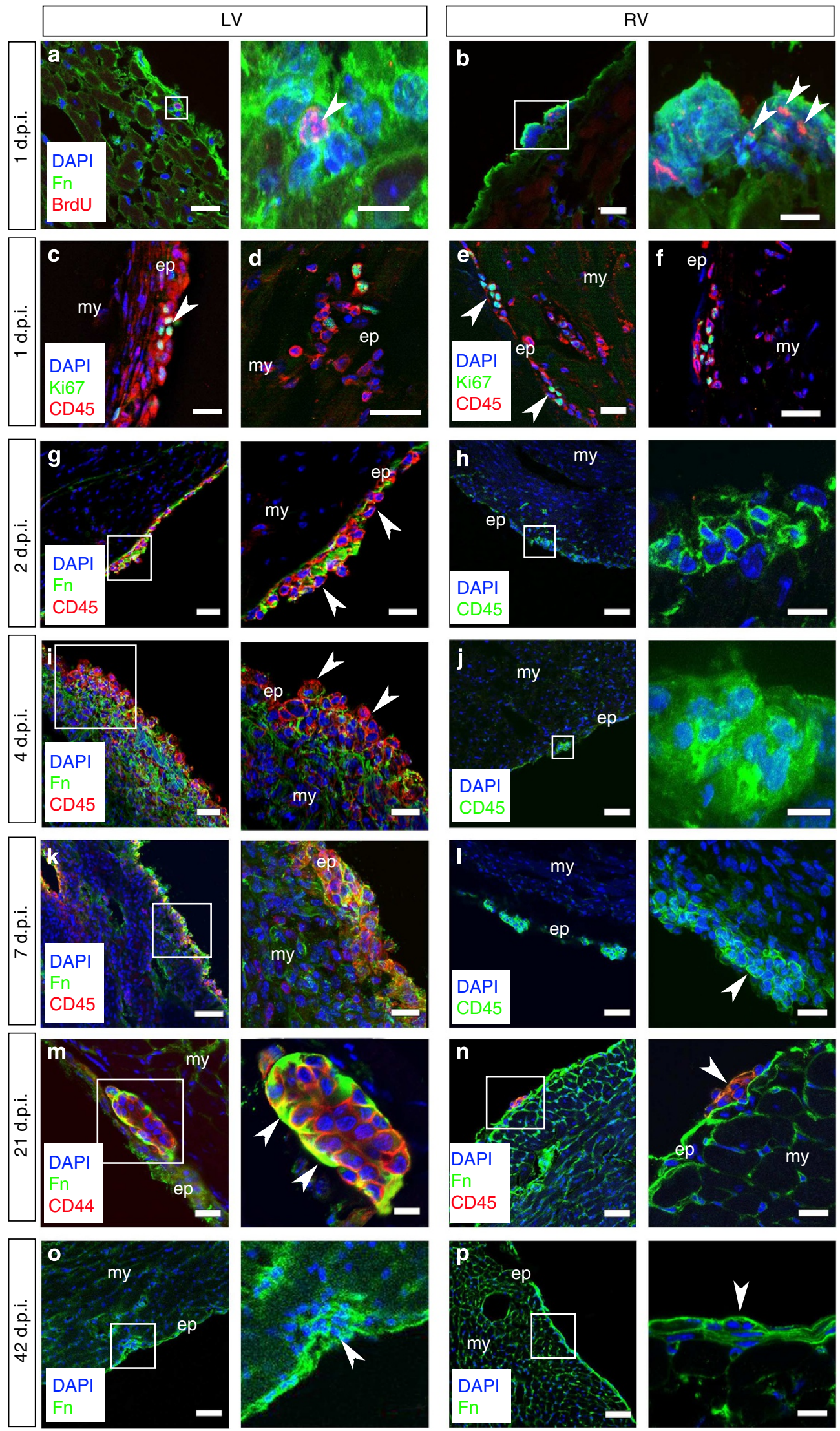


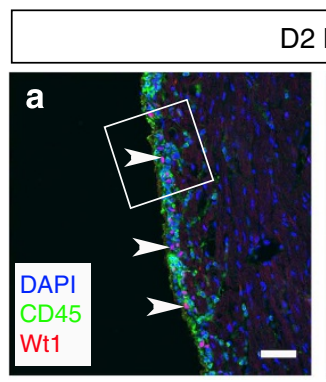

D2 MI
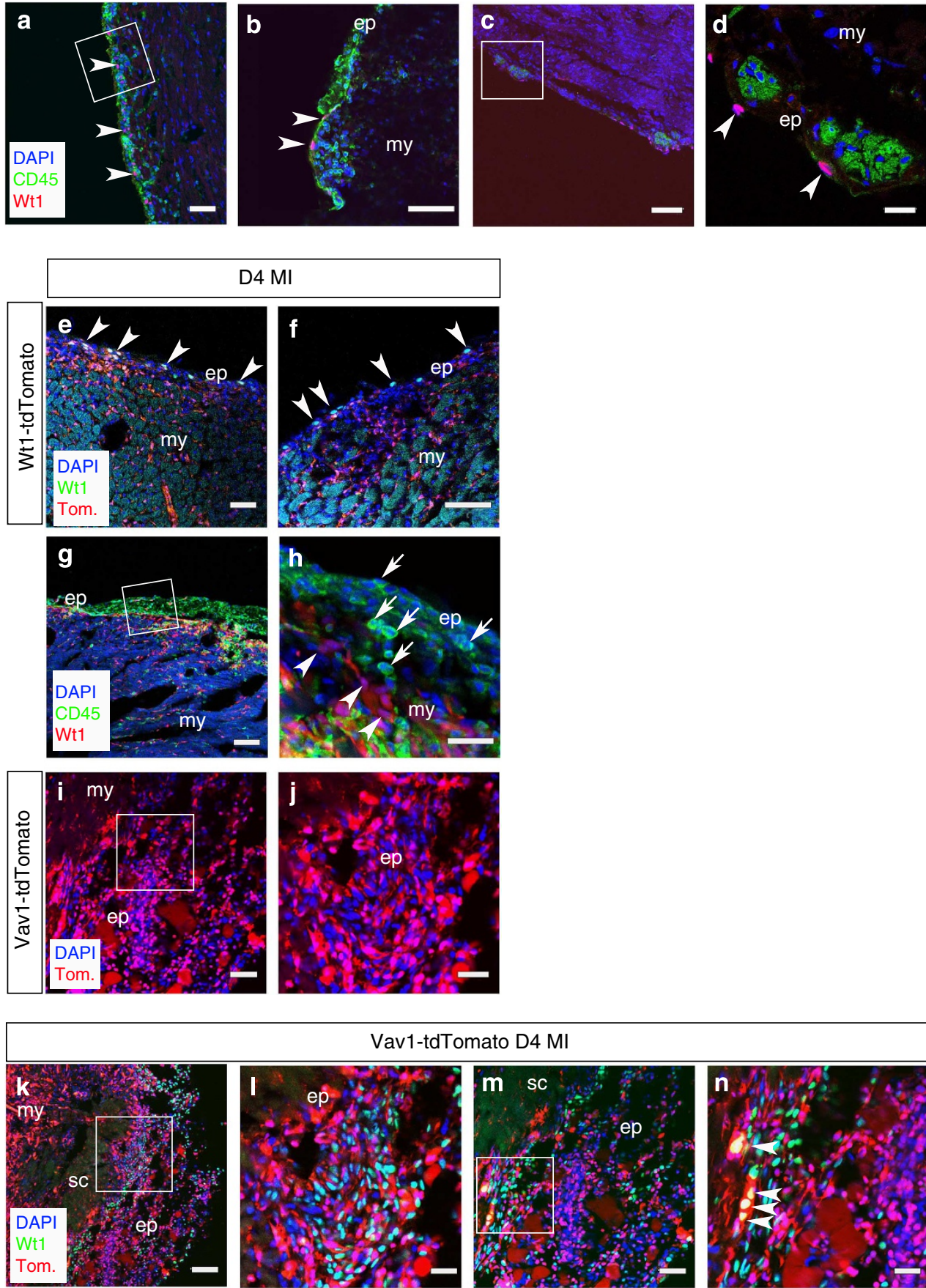

Figure 4 | Injury-activated Wt1 + epicardial cells are mutually exclusive from CD45/Vav1 + HCs in the expanded epicardium. At 2 days post MI (D2 MI), cells re-expressing Wt1 (highlighted by white arrowheads) are distinct from the CD45 + population residing in the expanding epicardium (a,b; white inset box in $\mathbf{a}$, shown at higher magnification in $\mathbf{b}$ ). At day 21 post MI (D21 MI), Wt1 cells reside proximal to the niche-like clusters (c,d; white inset box in c, shown at higher magnification in d). Wt1CreERT2;R26R-tdTomato labelling and Wt1-antibody staining revealed Wt1 $+/$ tdTomato + cells residing in the expanded epicardium and tdTomato-labelled derivatives within the underlying myocardium (e,f; arrowheads indicate double-positive $\mathrm{Wt1}+/$ tdTomato + cells) relative to an abundance of infiltrating CD45 + cells ( $\mathbf{g}, \mathbf{h}$; white inset box in $\mathbf{g}$ shown in higher magnification in $\mathbf{h}$; arrows in $\mathbf{h}$ indicate CD45 + cells and arrowheads indicate Wt1 + cells) and HCs labelled in a Vav1-Cre; R26R-tdTomato reporter line (i,j; white inset box in $\mathbf{i}$, shown at higher magnification in $\mathbf{j}$ ). At day 4 post MI (D4 MI), Wt1 and tdTomato are expressed in distinct cell populations with a few notable exceptions highlighted by white arrowheads (k-n; white inset boxes in $\mathbf{k}$ and $\mathbf{m}$ are highlighted at higher magnification in $\mathbf{I}$ and $\mathbf{n}$, respectively). ep, epicardium; my, myocardium; sc, scar. Scale bars, $50 \mu \mathrm{m},(\mathbf{a}, \mathbf{c}, \mathbf{e}, \mathbf{g}, \mathbf{i}) ; 100 \mu \mathrm{m}(\mathbf{k}, \mathbf{m}) ; 20 \mu \mathrm{m}(\mathbf{b}, \mathbf{d}) ; 30 \mu \mathrm{m}(\mathbf{f}, \mathbf{h}, \mathbf{j}, \mathbf{l}, \mathbf{n})$.

CD45 + epicardial cells arise from the haemagenic endothelium. To confirm a haematopoietic origin for the CD45 + population, we traced cells residing in the sub-epicardium using Vav1Cre; R26R-tdTomato mice. Vav1-Cre labels developmental haematopoietic progenitors arising from the yolk sac blood islands (Supplementary Fig. 6a,b) and the aorta-gonadmesonephros region (sites of primitive and definitive haematopoiesis, respectively; Supplementary Fig. 6c), the fetal liver 

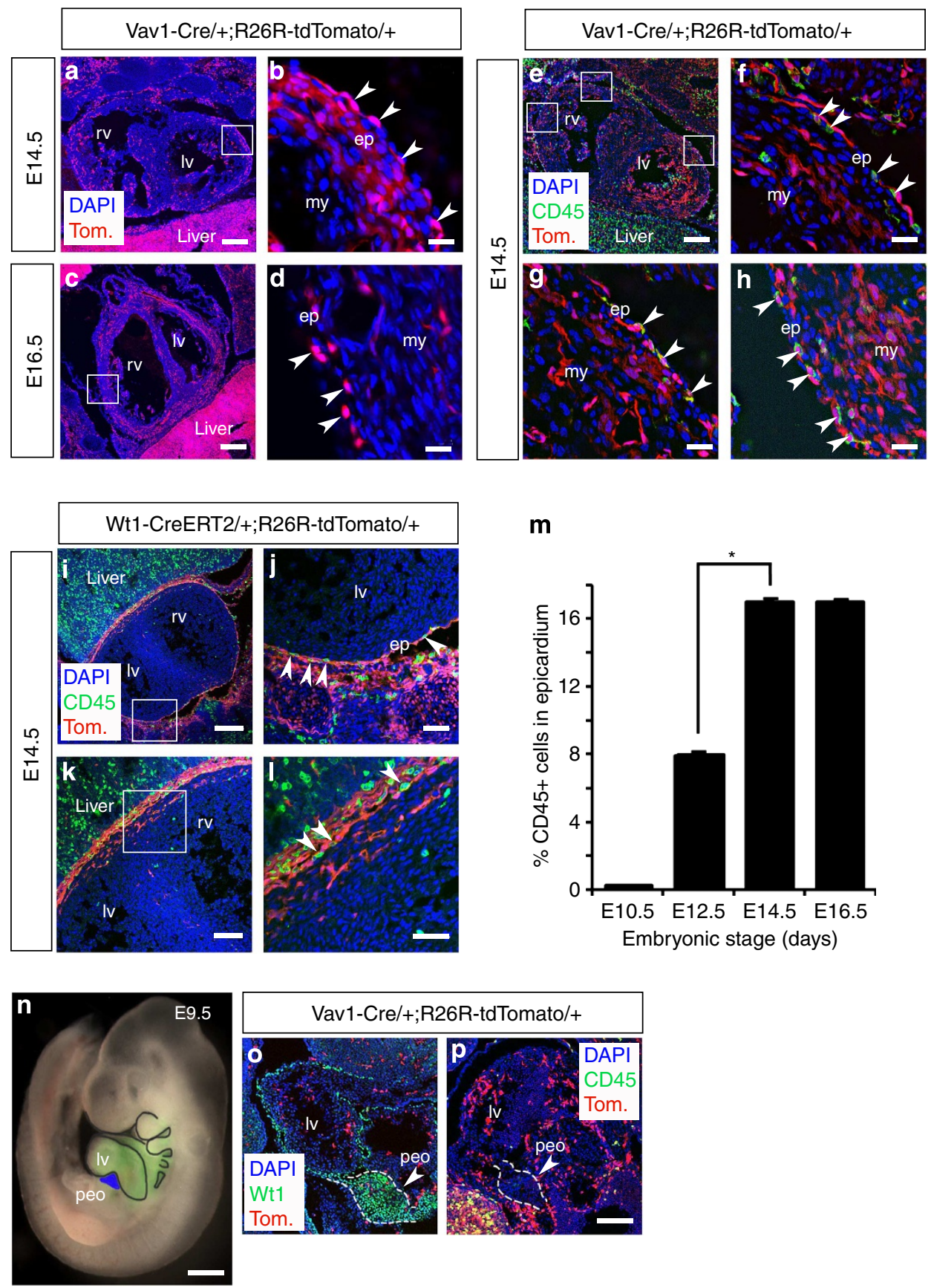

Figure 5 | Vav1 + /CD45 + HCs contribute to the developing epicardium and do not originate from the PEO. In Vav1-Cre;R26R-tdTomato embryonic hearts, tdTomato + (Vav1 + ) haematopoietic stem cells (HSCs) contribute to the developing epicardium from E12.5 to E16.5. At E14.5 (a,b) and E16.5 (c,d), tdTomato + (Tom) cells reside in the epicardium and underlying myocardium (white inset boxes in $\mathbf{a}$ and $\mathbf{c}$ are highlighted at higher magnification in $\mathbf{b}$ and $\mathbf{d}$, respectively; white arrowheads in $\mathbf{b}$ and $\mathbf{d}$ highlight tdTomato + cells specifically in the epicardium). At E14.5, further immunostaining for combined $\alpha$-CD45 + and tdTomato (e) revealed that all CD45 + cells residing within the developing epicardium were Vav1-tdTomato + (f-h; white arrowheads highlight double-positive cells), whereas Vav1-tdTomato + cells negative for CD45 + were localized within the underlying myocardium (f-h). The CD45 + $\mathrm{HCs}$ were distinct from the Wt1 + contribution as indicated by CD45 + immunostaining in conjunction with lineage tracing in WtCreERT2;R26R-tdTomato developing hearts at E14.5 (i-I; white inset boxes in $\mathbf{i}$ and $\mathbf{k}$ are highlighted at higher magnification in $\mathbf{j}$ and $\mathbf{I}$, respectively; white arrowheads in $\mathbf{j}$ and $\mathbf{I}$ highlight CD45 + cells specifically in the epicardium). The number of CD45 + cells that contributes to the epicardium increases from E10.5, coincident with formation of the epicardium, through to E16.5 with a significant increase between E12.5 and 14.5 (mean $\%$ CD $45+$ cells \pm s.e.m.; $P \leq 0.001 ; n=6$ hearts; $\mathbf{m}$ ). In Vav1-tdTomato-labelled embryos, tdTomato + cells do not contribute to the proepicardial organ (peo; indicated in blue in $\mathbf{n}$; rest of heart indicated in green via Nkx2.5Cre;R26R-EYFP) at E9.5 (o; peo is demarcated by Wt1 immunostaining) and CD45 + cells do not localize to the peo (p), suggesting tdTomato/CD45 + HCs that contribute to the forming epicardium arise from an alternate source, previously described as the haemagenic endothelium and/or fetal liver (Supplementary Fig. 6). ep, epicardium; Iv, left ventricle; my, myocardium; peo, proepicardial organ; rv, right ventricle. Scale bars, $200 \mu \mathrm{m}(\mathbf{a}, \mathbf{i}) ; 20 \mu \mathrm{m}(\mathbf{b}, \mathbf{d}, \mathbf{f}-\mathbf{h}) ; 400 \mu \mathrm{m}(\mathbf{c}, \mathbf{e}) ; 50 \mu \mathrm{m}(\mathbf{j}, \mathbf{l}) ; 100 \mu \mathrm{m}(\mathbf{i}, \mathbf{0}, \mathbf{p}) ; 600 \mu \mathrm{m}(\mathbf{n})$. All statistics are calculated by Students $t$-test; ${ }^{\star} P \leq 0.001$. 
(a second site of definitive haematopoiesis; Supplementary Fig. 6d,e), as well as adult pan-circulatory $\mathrm{HCs}^{17-19}$. Significant numbers of Vav1-tdTomato + cells were observed in the expanded sub-epicardium (Fig. $4 \mathrm{i}, \mathrm{j}$ ) by day 4 post MI and these were largely negative for $\mathrm{Wt1}$ protein expression aside from isolated examples of tdTomato $+/ \mathrm{Wt} 1+$ cells (Fig. 3k-n). The relative proportion of single tdTomato $+(\mathrm{Vav} 1+)$ versus single $\mathrm{Wt} 1+$ cells was $77.6 \pm 16.3 \%$ versus $22.4 \pm 4.8 \%$ (mean $\% \pm$ s.e.m.; $P \leq 0.001 ; n=6$ hearts; Student's $t$-test). Importantly, we excluded the endothelial markers, CD31 and Endomucin, from the CD45 + sub-population located within the epicardial and sub-epicardial regions at day 4 post MI, in support of an HC phenotype (Supplementary Fig. 7a-c).

Given the prevalence of CD45 + and Vav1 + cells in both the intact epicardium and in response to injury, we traced Vav1tdTomato + cells through developmental stages to determine whether there may be an embryonic haematopoietic contribution to the forming epicardium (Fig. 5). We observed tdTomato+ cells residing in the epicardium and underlying myocardium from E12.5, with a prominent contribution at both E14.5 (Fig. 5a,b) and E16.5 (Fig. 5c,d). The presence of HCs within the embryonic epicardium was confirmed by immunostaining for CD45 at E14.5 in Vav1-Cre;R26R-tdTomato mice (Fig. 5e-h), which revealed CD $45+/ \mathrm{m}$-tomato + cells integrated within the outer epicardial layer (Fig. 5f-h) that were negative for the endothelial cell marker CD31 (Supplementary Fig. 7d). Not all Vav1 + cells were CD45 +, consistent with the broader Vav1 + lineage ${ }^{17}$, but all CD45 + cells localized within the epicardium were $\mathrm{Vav1}+$, as evident in the lineage-traced hearts at E14.5 (Fig. 5f-h). At higher magnification (Fig. 5g), the CD45+/ tdTomato + cells were located on the apical surface of the heart, contiguous with the outer epicardium, suggesting they contributed intrinsically to the polarized epithelium. In contrast, immunostaining for CD45 in Wt1CreERT2;R26RtdTomato mice (Fig. 5i-l), revealed that the HCs were distinct from Wt1-tdTomato + epicardial cells (Fig. 51). The incidence of CD45 + cells residing in the epicardium significantly increased from $7.9 \pm 0.01 \%$ at E12.5 to a maximum of $17.0 \pm 1.2 \%$ at E14.5 $(P \leq 0.001 ; n=6$ hearts; Student's $t$-test; Fig. $5 \mathrm{~m})$. To exclude the possibility that the CD45 + cells might have arisen from the PEO (Fig. 5n), we stained Vav1-tdTomato mice with $\alpha$-CD45 and observed that both tdTomato and CD45 were not expressed within the $\mathrm{Wt} 1+$ PEO (Fig. 5o,p). In addition, lineage tracing with Gata5Cre;R26R-tdTomato and Wt1CreERT2;R26RtdTomato mice revealed that neither Gata5 + nor Wt1+ epicardial lineages gave rise to CD45 cells (Supplementary Fig. 8). In contrast, a proportion of Gata5-tdTomato + cells $(<10 \%)$ were also CD44+, consistent with a mesenchymal-like cell origin from the $\mathrm{PEO}^{9}$. These data suggest that HCs contribute to the forming epicardium during development and arise from a Vav1 + haemogenic (haemangiogenic) source, which is distinct from the PEO.

Bone marrow-derived HCs integrate into the adult epicardium. To explore a potential haematopoietic contribution to the epicardium of the intact heart during adult homeostasis, we carried out cell transplantation of either labelled bone marrow (BM) from $\beta$-actin-Cre;R26R-EYFP or HCs from Vav1-Cre;R26RtdTomato donors into lethally or sub-lethally irradiated unlabelled hosts (Supplementary Fig. 9a,b). Ionizing radiation targets mitotically active cells, which in the intact adult epicardium are likely to be represented by the previously described CD44+ cCFU-Fs $^{9}$. Haematopoietic reconstitution occurs in mouse in waves, with transient reconstituting cells detectable after 2-4 weeks, but which disappear after 8-10 weeks, and longer-term reconstituting cells that contribute at least 6 months after transplantation $^{20}$. Thus, a 6-month follow-up herein was employed to negate the possibility of $\mathrm{HC}$ influx due to irradiation-induced inflammation and, consequently, to investigate potential long-term (homeostatic) BM contribution and epicardial cell turnover. Following irradiation, we were unable to detect either CD44 + cells, consistent with ablation of the cCFU-Fs as previously described ${ }^{9}$ or host CD45 + cells, even after 6 months. YFP + BM cells were observed in the epicardium and sub-epicardial region after 2 months, residing both within the single epicardial cell layer overlying the myocardium and epicardial cell clusters (Fig. 6a-d). YFP + cells were also observed to traffic into adult epicardial clusters at 2 months (Supplementary 10a,b). At 6 months, a YFP + contribution was still evident in the epicardium with some contribution to the adjacent myocardium (Fig. 6e-h). Quantitation revealed the overall numbers of YFP + BM cells significantly increased in the epicardium over the 4-month duration of the analyses $(P \leq 0.001$; $n=6$ hearts; Student's $t$-test; Fig. 6i). Although early on, following host $\gamma$-irradiation, donor BM-derived cells may infiltrate due to radiation-induced injury and inflammation, the significant $\mathrm{YFP}+\mathrm{BM}$ presence at 6 months represents an injury-independent contribution. Podoplanin immunostaining confirmed the localization of YFP + BM derivatives to the epicardium at both 2 and 6 months (Fig. 6j,n); although there was significant variation in the abundance of transplanted cells depending on location within the chambers (compare Fig. 6k,m with Fig. $6 \mathrm{n}, \mathrm{q})$, after 2 and 6 months. The YFP + cells were all $\mathrm{CD} 45+$ indicative of the HC compartment of the BM inoculum (Fig. 6k,o). The majority of those residing within the epicardium itself $(>90 \%)$ were negative for the monocyte marker Ly-6C (Fig. 61,p); however, a more detailed characterization of their immuno-phenotype revealed a minor subpopulation $(<10 \%)$ of transplanted tomato $+/ \mathrm{CD} 45+$ cells, which were positive for the macrophage marker F4/80 (Supplementary Fig. 11a,b); in contrast, none of the transplanted cells co-stained for the neutrophil marker myeloperoxidase (Supplementary Fig. 11c). Evidence of subsequent YFP + BM cell differentiation within the myocardium was observed by virtue of cells co-staining for the pericyte marker NG2 + (Fig. 6m,q; Supplementary Fig. 12a). Further analyses on the lineage potential of the transplanted BM cells at 2 months revealed co-staining of YFP + cells with an additional pericyte marker CD146 (ref. 21 (Supplementary Fig. 12b) and the exclusion of the fibroblast marker PDGFR $\beta$ Supplementary Fig. 12c,d).

Transplanted Vav1 + CD45 + cells become depleted with age. The BM findings were supported by transplantation with lineagenegative $\mathrm{HCs}$ as labelled by Vav1-Cre;R26R-tdTomato. Tomato + cells were observed in the epicardium at 2 months as demarcated by podoplanin (Fig. 6r) and were localized into clusters (Supplementary Fig. 10b). These cells were CD45 + , and consistent with the whole $\mathrm{BM} \mathrm{YFP}+$ transplantation data the majority were Ly-6C - (Fig. 6s,t), of which a proportion had differentiated into $\mathrm{NG} 2+$ pericytes located in the underlying myocardium (Fig. 6u), consistent with the previous observations for whole BM. Tomato + cells post-BM transplantation either residing within the epicardial or underlying myocardium were also negative for the endothelial cell markers CD31 and Endomucin (Supplementary Fig. 7e-h). We subsequently investigated the status of the CD45 + compartment in older animals, given that aging is proposed to act as a negative modifier of cellular efficacy (reviewed in ref. 22). A time course from post-natal day 7 (P7) through to 18 months suggested the epicardium is turned over via HC contribution, with increasing recruitment of CD45+ 

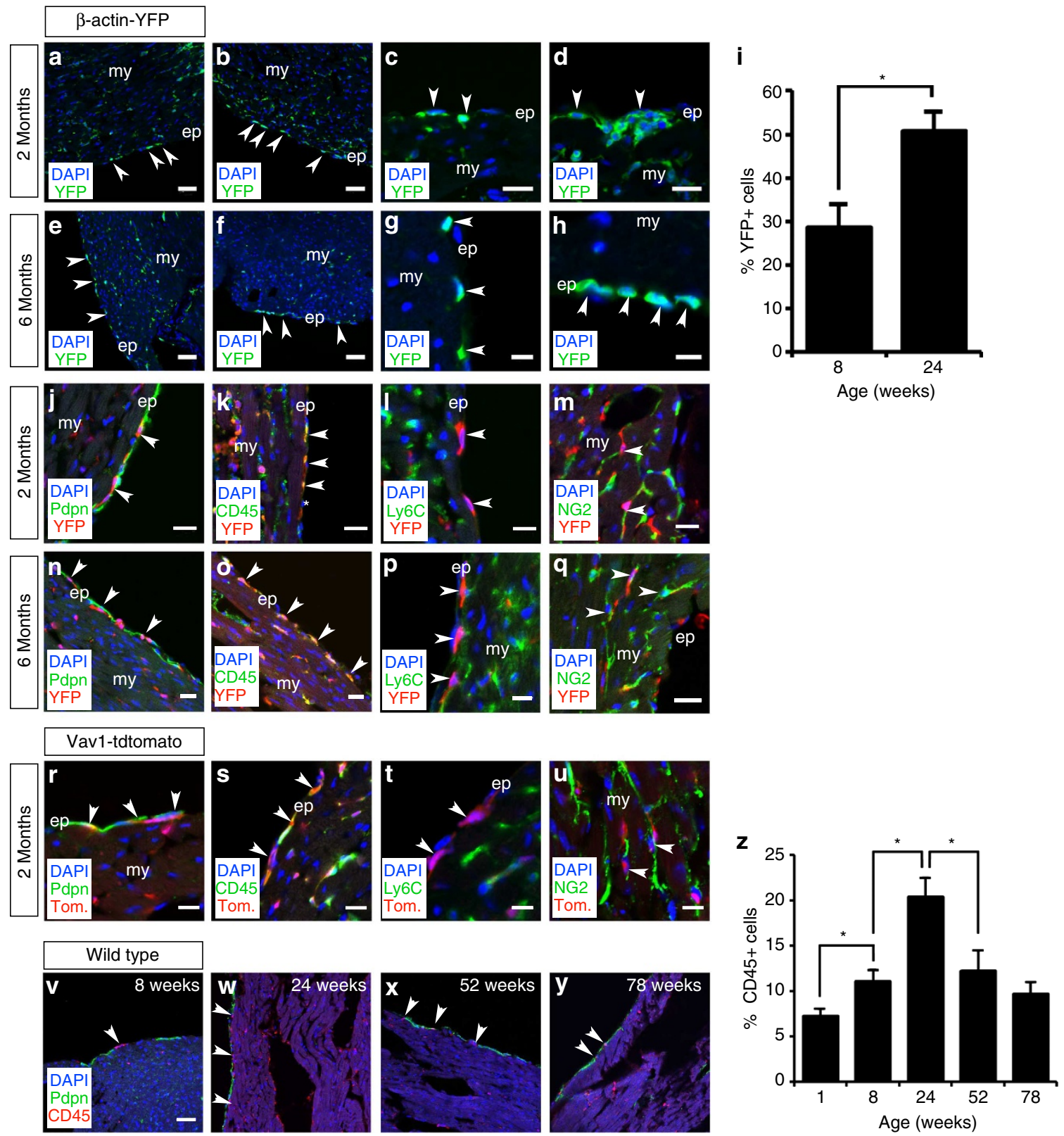

Figure 6 | Bone marrow transplantation reveals a haematopoietic contribution to the adult epicardium that alters with age. Two months after whole bone marrow (BM) transplantation of $\beta$-actin-Cre;R26R-EYFP labelled BM into lethally irradiated unlabelled hosts (Supplementary Fig. 9a), YFP + cells are localized to the adult epicardium (a,b; as highlighted by white arrowheads) and the YFP + BM cells contribute to epicardial clusters (c,d). The YFP + cells persist at 6 months post transplantation and are localized to the epicardium (e-h; as highlighted by white arrowheads) and there was a significant increase in epicardial YFP + cells over the intervening 4 months (mean \%YFP + cells \pm s.e.m.; $P \leq 0.001 ; n=6$ hearts; i). At 2 and 6 months, podoplanin immunostaining confirmed the epicardial localization of YFP + BM cells $(\mathbf{j}, \mathbf{n})$ and these were CD45+ (k,o; white arrowheads; white asterisk highlights a YFP +/CD45 - cell), suggesting they represented the haematopoietic compartment of the whole BM inoculum, but were negative for Ly6C, thus excluding the mature myeloid lineage (I,p; white arrowheads indicate YFP + cells in red which do not co-express Ly6C in green). A sub-population of YFP + BM cells within the underlying myocardium expressed NG2, suggesting they had differentiated into pericytes (m,q; highlighted by white arrowheads). Two months after transplantation of flow-sorted Vav1 + cells, from Vav1-Cre;R26R-tdTomato-labelled bone marrow into lethally irradiated unlabelled hosts (Supplementary Fig. 9b), tdTomato + cells are localized to the podoplanin + adult epicardium (r; as highlighted by white arrowheads). The tdTomato + BM cells were CD45 + (s; white arrowheads), confirming them as representative of the haematopoietic compartment of the whole BM inoculum. The tdTomato + donor cells were negative for Ly6C, thus excluding the mature myeloid lineage ( $\mathbf{t}$; white arrowheads indicate tdTomato + cells in red, which do not co-express Ly6C in green). A sub-population of tdTomato + BM cells within the underlying myocardium expressed NG2, suggesting they had differentiated into pericytes ( $\mathbf{u}$; highlighted by white arrowheads). The number of CD45 + cells increased in the adult epicardium with age up to 24 weeks $(\mathbf{v}, \mathbf{w})$ but decreased thereafter as measured at 52 and 78 weeks $(\mathbf{x}, \mathbf{y})$. The increases between 1 and 8 , and 8 and 24 weeks were significant, as was the decrease between 24 and 52 weeks (mean \% CD45 + cells \pm s.e.m. of $n=3$ hearts, 6 sections analysed per heart; $P \leq 0.001 ; \mathbf{z}$ ), suggesting an increased haematopoietic epicardial reserve with adulthood to maturity but depletion or impaired replenishment with advanced age (12 and 18 months). ep, epicardium; my, myocardium. Scale bars, $50 \mu \mathrm{m}(\mathbf{a}, \mathbf{b}, \mathbf{e}, \mathbf{f}, \mathbf{v}-\mathbf{y}) ; 20 \mu \mathrm{m}(\mathbf{c}, \mathbf{d}, \mathbf{j}, \mathbf{k}-\mathbf{0} \mathbf{0}, \mathbf{q}-\mathbf{s}, \mathbf{u}) ; 10 \mu \mathrm{m} ;(\mathbf{g}, \mathbf{h}) ; 10 \mu \mathrm{m}(\mathbf{i}, \mathbf{p}, \mathbf{t})$. All statistics are calculated by Student's t-test; ${ }^{\star} P \leq 0.001$. 
cells between P7 and 6 months (Fig. 6v-y). In older mice, the number of $\mathrm{CD} 45+$ cells was significantly reduced from $20.3 \pm 2.1 \%$ at 6 months down to $12.2 \pm 2.2 \%$ at 12 months and $9.67 \pm 1.3 \%$ at 18 months (mean \pm s.e.m. of $n=3$ hearts, 6 sections analysed per heart; $P \leq 0.001$; Student's $t$-test; Fig. $6 \mathrm{z}$ ), suggesting that either $\mathrm{HC}$ contribution to the epicardium is compromised with age or the resident $\mathrm{CD} 45+$ population is depleted over time.

\section{Discussion}

The characterization of the adult epicardium as a complex anatomical structure is in stark contrast to the widely held view of the epicardium as a simple mesothelium and single cell layer epithelium. The adult epicardial topology contained features associated with a canonical stem cell niche, as have been characterized in the BM, skin (hair follicle bulge), intestine (villi and crypts) and subventricular zone of the brain ${ }^{23-26}$. Most notably, the existence of clusters of cells, within the epicardium, encased by basement membrane and with associated vascular support meets several anatomical niche criteria (reviewed in ref. 27). In the adult heart, cell turnover and renewal based on stem cell deployment has been demonstrated ${ }^{9,28}$; yet, occupancy of a cardiogenic stem cell niche ${ }^{29}$ remains to be determined ${ }^{30,31}$. Whether the epicardium can be regarded as a classical niche requires functional disruption of the 'microenvironment' described, so as to directly affect the resident stem/progenitor cell population in terms of homing, multipotency or differentiation of progeny during homeostasis or injury. These represent ongoing, but technically challenging, experiments that lie outside the scope of the current study.

The 'niche-like' structure is formed during a critical post-natal window when the inherent regenerative capacity of the heart is lost within the first week after birth ${ }^{12,32}$. This may reflect the limited reparative capacity of the maturing heart, at least in part, via the constraint of epicardial cells within the ECM (Supplementary Movie 1). Following myocardial injury, an initial breakdown of epicardial basement membrane was accompanied by release of constituent cells and subsequent reformation via assembly of ECM. The ECM plays a key role in a niche setting, as it is thought to 'protect' the progenitor compartment from depletion while simultaneously protecting the host tissue from overproliferation. Although the resident cells remain in a state of quiescent under normal conditions, the ECM is also important for transmitting signals to induce self-renewal and/or differentiation during physiological stress or in response to injury (reviewed in ref. 33). The resident cellular compartments in the epicardium consist of both $\mathrm{CD} 44+$ mesenchymal-like stem cells (cCFU-Fs), previously demonstrated to be of epicardial origin $^{9}$, and a novel BM-derived $\mathrm{CD} 45+$ haematopoietic population, which appeared to participate in homeostatic EPDC turnover with age. Both cell types are incorporated into the clusters irrespective of origin during the first week of life and the $\mathrm{CD} 45+$ population is subsequently replenished from BM. Although tissue monocytes of $\mathrm{BM}$ origin are known to migrate into almost every solid organ of the body, including the adult heart (reviewed in ref. 34), an HC contribution to the epicardium represents a novel finding. The contribution to adult epicardium by HCs was injury independent, with long-term reconstitution of donor HCs following BM transplantation well beyond any potential inflammatory response arising from host irradiation. Although we were unable to definitively discriminate the response of a resident $\mathrm{CD} 45+/ \mathrm{Vav} 1+$ population from $\mathrm{BM}$ infiltration during injury, the remodelling of the ECM surrounding the cell clusters and resident CD45 + cell proliferation was distinct from an influx of $\mathrm{BM} \mathrm{HCs} \mathrm{post} \mathrm{MI.} \mathrm{Moreover,} \mathrm{the} \mathrm{long-term}$ reconstitution of $\mathrm{BM} / \mathrm{Vav1}+$ transplanted cells suggested the $\mathrm{BM}$ can act as a source of replenishment of epicardial HCs, which in turn are then able to respond to injury independently of an acute $\mathrm{BM}$ response.

Previously, whole BM transplantation revealed that engraftment of BM-derived cells occurred as part of normal cardiac valve function, setting a precedent for a BM contribution to cardiovascular homeostasis per $s e^{35}$. Our study supports a role for $\mathrm{BM}$ in adult heart homeostasis and the HC turnover, contrasts with the current steady-state view of a single source of epicardium maintained throughout adulthood; this in turn reinforces the concept that the adult mammalian heart should no longer be considered a post-mitotic organ ${ }^{36-38}$.

CD45 + HCs were labelled by a Vav1-Cre-induced reporter, which, when subsequently traced through developmental stages, revealed a novel contribution to the embryonic epicardium from the haemagenic endothelium or fetal liver ${ }^{18,19}$. A distinct sub-population of HCs was recently characterized within the developing heart as arising from the endocardial lineage, and contributing an additional reservoir for definitive haematopoiesis ${ }^{39}$. In contrast, we report here the integration of $\mathrm{CD} 45+\mathrm{HCs}$ into the developing epicardium, arising from classical sites of both primitive (yolk sac blood islands) and definitive (aorta-gonad-mesonephros, fetal liver) haematopoiesis within the embryo. Notably, the CD45 + /Vav1 + population was entirely distinct from Wt1-expressing cells of the PEO. Cellular heterogeneity within the epicardium has been reported in previous studies, which focused on the lineage composition of the PEO, as the canonical source of epicardium progenitors $9,40,41$. Although a number of sub-compartments within the PEO itself have been well characterized, notably those labelled as $\mathrm{Wt} 1+$ and Tbx18+, as well as partially overlapping Sema3D + and Scleraxis + sub-populations ${ }^{40}$, a novel source of epicardial cells of alternate embryological origin has not hitherto been reported. Our findings challenge current dogma that the epicardium arises exclusively from the transient, mesoderm-derived PEO, located at the inflow region of the developing heart ${ }^{5}$.

Understanding the developmental biology of the epicardium is instrumental for developing novel cell therapies for heart regeneration. We and others have previously demonstrated that the adult epicardium, can be reactivated, via induction of an embryonic gene programme, to act as a potential resident cell source, which can be tapped to reconstitute damaged heart tissue $^{2-4,7,42}$. This potential of the adult epicardium was realized entirely in the context of a homogeneous $\mathrm{Wt} 1+$ epicardial cell response. The integral contribution of $\mathrm{CD} 45+\mathrm{HCs}$ to both embryonic and adult epicardium, and the turnover of a BM-derived HC source during adult homeostasis, should yield important insight into the potential for both self-renewal and resident cell-based repair within the heart. It will be of interest to determine whether the $\mathrm{HC}$ contribution to the epicardium and complex tertiary structure of constraining ECM identified here in mouse translates to humans, and whether it might be targeted for activation and contribution to tissue regeneration following ischaemic heart disease.

\section{Methods}

Generation of epicardial trace mice. Wt1 ${ }^{\mathrm{CreERT} 2 /+}$, Gata $5^{\mathrm{Cre} /}+$ and $\mathrm{Vav1}{ }^{\mathrm{Cre} /+}$; $\mathrm{R} 26 \mathrm{R}^{\text {tdTomsto/ + mice were generated crossing Rosa26R-tdTomato }}{ }^{43}$ reporter mice with Wt1 CreERT2/+ (ref. 44), Gata5 ${ }^{\mathrm{Cre} /+}$ (ref. 45) and Vav1 ${ }^{\mathrm{Cre} /}+$ (ref. 46) mice, and genotyping as previously described. Wt1 expression in the developing epicardium was achieved through double intraperitoneal tamoxifen injections. Toxic effects of tamoxifen were countered by co-injection of progesterone. Pregnant dams received two 2-mg tamoxifen injections and simultaneous $1 \mathrm{mg}$ progesterone injections at E9 and E11. Embryos were collected at specific time points and prepared for immunofluorescence. Images were obtained using a using Zeiss LSM 710 confocal microscope equipped with argon and helium neon lasers using $\times 20, \times 40$ and $\times 63 / 1.4$ (oil immersion) objectives. All animal experiments 
were carried out according to the UK Home Office project licences PPL30/2987 Compliant with the UK Animals (Scientific Procedures) Act 1986 and approved by the University College London Biological Services Ethical Review Process. Animal husbandry at UCL Biological Services was in accordance with the UK Home Office Certificate of Designation.

Immunodetection. Immunofluorescence was performed on cryosections of embryonic, uninjured and post-MI hearts using standard protocols with the following antibodies: green fluorescent protein (GFP; Clontech and Abcam, which detect EYFP), Fibronectin, CD44, CD45, BrdU, Hyaluronic acid, Ly-6C and Wt1 (all from Abcam), podoplanin (Novus Europe), Collagen IV (AbD Serotec), NG2 (Chemicon) and CD29 (Millipore). To rule out the possibility of autofluorescence accounting for the detection of either GFP or yellow fluorescent protein (YFP) protein expression, sections through the LV were stained with a polyclonal anti-GFP antibody (which detects both fluorescent proteins). The specificity of the anti-GFP antibody was ascertained by immunofluorescence on non-primed, intact hearts, which detected neither labelled cells in the epicardial region, nor their derivatives (no signal)

To detect BrdU-positive nuclei, sections were treated with $2 \mathrm{~N} \mathrm{HCl}$ for $30 \mathrm{~min}$ at room temperature $\left(22^{\circ} \mathrm{C}\right)$ to denature the DNA, and neutralized in $0.1 \mathrm{M}$ sodium borate $\mathrm{pH} 8.5$ for $12 \mathrm{~min}$ before incubation with the anti-BrdU antibody. Owing to the destruction of cellular antigens resulting from acid treatment, these steps were performed after the incubation with antibodies to GFP and $\mathrm{S} \alpha \mathrm{A}$. Images were acquired using a Zeiss LSM 710 confocal microscope equipped with argon and helium neon lasers using $\times 20, \times 40$ and $\times 63 / 1.4$ (oil immersion) objectives.

mTomato fluorescent protein used to test the Cre-lox recombination of Gata5, $W t 1$ and Vav1 Cre driver strains was visualized directly without secondary staining protocols at its emission wavelength of $581 \mathrm{~nm}$.

MI surgery. Mice were housed and maintained in a controlled environment. All surgical and pharmacological procedures were performed in accordance with the Animals (Scientific Procedures) Act 1986, (Home Office, UK). MI was induced in isoflurane-anaesthetized mice by permanent ligation of the left anterior descending artery. On recovery, animals received intraperitoneal injection of BrdU $\left(80 \mathrm{mg} \mathrm{kg}^{-1}\right.$; Invitrogen) if required. Further injections were administered at 2,4 and $8 \mathrm{~h}$ post surgery. Hearts were harvested at 2, 4, 7, 21 and 42 days after ligation and bisected transversely midway through the scar and fixed in $4 \%$ paraformaldehyde for cryosectioning and immunostaining analyses.

BM collection. All mice were culled before tissue dissection in accordance with the Home Office regulation. Hind limbs were dissected to isolate the femur and knee joint. Femurs were flushed using a 23-G needle and 1-ml syringe (both from Becton Dickinson) with PBS, and cells were collected and kept on ice. Cells were then filtered using a $70-\mu \mathrm{m}$ cell strainer (Becton Dickinson) and centrifuged at $200 \mathrm{~g}$ for $5 \mathrm{~min}$. Five millilitres of Red Cell Lysis Buffer was used to resuspend the pellet and incubated for $3 \mathrm{~min}$ before centrifuging at $200 \mathrm{~g}$ for $5 \mathrm{~min}$ to pellet the cells. Cells were then used for RNA extraction or transplantation.

BM irradiation and transplantation. Mice are housed and maintained in a controlled environment. All procedures are performed in accordance with the Animals (Scientific Procedures) Act 1986, (Home Office, UK). Mice were sublethally $(6 \mathrm{~Gy})$ irradiated or lethally irradiated, using split dose $(4+5 \mathrm{~Gy})$ radiation, $24 \mathrm{~h}$ before cell transfer.

Donor mice were culled before tissue dissection in accordance with the Home Office regulation. Hind limbs were dissected to isolate the femur and tibia bones. Single-cell suspensions were isolated in Hepes-buffered HBSS and either transplanted directly into recipient mice or lineage-negative cells were first purified using the mouse Lineage Cell Depletion kit (Miltenyi Biotec). In total, $1 \times 10^{7}$ or $2.5 \times 10^{5}$ lineage-negative cells were injected via the lateral tail vein.

\section{References}

1. Hosoda, T. et al. Clonality of mouse and human cardiomyogenesis in vivo. Proc. Natl Acad. Sci. USA 106, 17169-17174 (2009).

2. Smart, N. et al. Thymosin beta4 induces adult epicardial progenitor mobilization and neovascularization. Nature 445, 177-182 (2007).

3. Smart, N. et al. De novo cardiomyocytes from within the activated adult heart after injury. Nature 474, 640-644 (2011).

4. Zhou, B. et al. Adult mouse epicardium modulates myocardial injury by secreting paracrine factors. J. Clin. Invest 121, 1894-1904 (2011)

5. Riley, P. R. An epicardial floor plan for building and rebuilding the mammalian heart. Curr. Topics Dev. Biol. 100, 233-251 (2012).

6. Olivey, H. E. \& Svensson, E. C. Epicardial-myocardial signaling directing coronary vasculogenesis. Circ. Res. 106, 818-832 (2010).

7. Bock-Marquette, I. et al. Thymosin beta4 mediated PKC activation is essential to initiate the embryonic coronary developmental program and epicardial progenitor cell activation in adult mice in vivo. J. Mol. Cell Cardiol. 46, 728-738 (2009)
8. Pittenger, M. F. et al. Multilineage potential of adult human mesenchymal stem cells. Science 284, 143-147 (1999).

9. Chong, J. J. et al. Adult cardiac-resident MSC-like stem cells with a proepicardial origin. Cell Stem Cell 9, 527-540 (2011).

10. Kawaguchi, M., Bader, D. M. \& Wilm, B. Serosal mesothelium retains vasculogenic potential. Dev. Dyn. 236, 2973-2979 (2007).

11. Chen, T. H. et al. Epicardial induction of fetal cardiomyocyte proliferation via a retinoic acid-inducible trophic factor. Dev. Biol. 250, 198-207 (2002).

12. Porrello, E. R. et al. Transient regenerative potential of the neonatal mouse heart. Science 331, 1078-1080 (2011).

13. Fuchs, E. \& Horsley, V. Ferreting out stem cells from their niches. Nat. Cell Biol. 13, 513-518 (2011)

14. Braitsch, C. M., Kanisicak, O., van Berlo, J. H., Molkentin, J. D. \& Yutzey, K. E. Differential expression of embryonic epicardial progenitor markers and localization of cardiac fibrosis in adult ischemic injury and hypertensive heart disease. J. Mol. Cell Cardiol. 65, 108-119 (2013).

15. van Wijk, B., Gunst, Q. D., Moorman, A. F. \& van den Hoff, M. J. Cardiac regeneration from activated epicardium. PLoS ONE 7, e44692 (2012).

16. Nahrendorf, M. et al. The healing myocardium sequentially mobilizes two monocyte subsets with divergent and complementary functions. J. Exp. Med. 204, 3037-3047 (2007)

17. Georgiades, P. et al. VavCre transgenic mice: a tool for mutagenesis in hematopoietic and endothelial lineages. Genesis 34, 251-256 (2002).

18. Chen, M. J., Yokomizo, T., Zeigler, B. M., Dzierzak, E. \& Speck, N. A. Runx1 is required for the endothelial to haematopoietic cell transition but not thereafter. Nature 457, 887-891 (2009).

19. Ruiz-Herguido, C. et al. Hematopoietic stem cell development requires transient Wnt/beta-catenin activity. J. Exp. Med. 209, 1457-1468 (2012).

20. Morrison, S. J. \& Weissman, I. L. The long-term repopulating subset of hematopoietic stem cells is deterministic and isolatable by phenotype. Immunity 1, 661-673 (1994).

21. Chen, C. W. et al. Human pericytes for ischemic heart repair. Stem Cells 31, 305-316 (2013).

22. Dimmeler, S. \& Leri, A. Aging and disease as modifiers of efficacy of cell therapy. Circ. Res. 102, 1319-1330 (2008).

23. Goldberg, J. S. \& Hirschi, K. K. Diverse roles of the vasculature within the neural stem cell niche. Regen. Med. 4, 879-897 (2009).

24. Zhang, J. et al. Identification of the haematopoietic stem cell niche and contro of the niche size. Nature 425, 836-841 (2003).

25. Benitah, S. A., Frye, M., Glogauer, M. \& Watt, F. M. Stem cell depletion through epidermal deletion of Rac1. Science 309, 933-935 (2005).

26. Sato, T. et al. Paneth cells constitute the niche for Lgr5 stem cells in intestinal crypts. Nature 469, 415-418 (2011).

27. Spradling, A., Drummond-Barbosa, D. \& Kai, T. Stem cells find their niche. Nature 414, 98-104 (2001).

28. Kajstura, J. et al. Cardiomyogenesis in the adult human heart. Circ. Res. 107, 305-315 (2010).

29. Urbanek, K. et al. Stem cell niches in the adult mouse heart. Proc. Natl Acad. Sci. USA 103, 9226-9231 (2006).

30. Balmer, G. M. \& Riley, P. R. Harnessing the potential of adult cardiac stem cells: lessons from haematopoiesis, the embryo and the niche. J. Cardiovasc. Trans. Res. 5, 631-640 (2012).

31. Christalla, P., Hudson, J. E. \& Zimmermann, W. H. The cardiogenic niche as a fundamental building block of engineered myocardium. Cells Tissues Organs 195, 82-93 (2012).

32. Mahmoud, A. I. et al. Meis1 regulates postnatal cardiomyocyte cell cycle arrest Nature 497, 249-253 (2013).

33. Gattazzo, F., Urciuolo, A. \& Bonaldo, P. Extracellular matrix: a dynamic microenvironment for stem cell niche. Biochim. Biophys. Acta. doi:10.1016/ j.bbagen.2014.01.010.

34. Nahrendorf, M. \& Swirski, F. K. Monocyte and macrophage heterogeneity in the heart. Circ. Res. 112, 1624-1633 (2013).

35. Hajdu, Z. et al. Recruitment of bone marrow-derived valve interstitial cells is a normal homeostatic process. J. Mol. Cell Cardiol. 51, 955-965 (2011)

36. Bergmann, O. et al. Evidence for cardiomyocyte renewal in humans. Science 324, 98-102 (2009)

37. Senyo, S. E. et al. Mammalian heart renewal by pre-existing cardiomyocytes. Nature 493, 433-436 (2013).

38. Mollova, M. et al. Cardiomyocyte proliferation contributes to heart growth in young humans. Proc. Natl Acad. Sci. USA 110, 1446-1451 (2013).

39. Nakano, H. et al. Haemogenic endocardium contributes to transient definitive haematopoiesis. Nat. Commun. 4, 1564 (2013).

40. Katz, T. C. et al. Distinct compartments of the proepicardial organ give rise to coronary vascular endothelial cells. Dev. Cell 22, 639-650 (2012).

41. Acharya, A. et al. The bHLH transcription factor Tcf21 is required for lineage-specific EMT of cardiac fibroblast progenitors. Development 139, 2139-2149 (2012). 
42. Bollini, S., Smart, N. \& Riley, P. R. Resident cardiac progenitor cells: at the heart of regeneration. J. Mol. Cell Cardiol. 50, 296-303 (2010).

43. Madisen, L. et al. A robust and high-throughput Cre reporting and characterization system for the whole mouse brain. Nat. Neurosci. 13, 133-140 (2010).

44. Zhou, B. et al. Epicardial progenitors contribute to the cardiomyocyte lineage in the developing heart. Nature 454, 109-113 (2008).

45. Merki, E. et al. Epicardial retinoid X receptor alpha is required for myocardial growth and coronary artery formation. Proc. Natl Acad. Sci. USA 102, 18455-18460 (2005).

46. de Boer, J. et al. Transgenic mice with hematopoietic and lymphoid specific expression of Cre. Eur. J. Immunol. 33, 314-325 (2003).

\section{Acknowledgements}

We thank Bertrand Vernay at the UCL-ICH Light Microscopy Core Facility for technical assistance with imaging and Cynthia Andoniadou for early embryo dissections. This work was generously supported by a Wellcome Trust PhD studentship 089592/Z/09/A (G.M.B.) and British Heart Foundation programme grant RG/ 08/003/25264 (P.R.R.).

\section{Author contributions}

G.M.B. carried out the experiments, analysed the data and assisted in compiling figures; S.B. carried out immunostaining analyses, analysed data and provided MI heart injury samples; K.N.D. provided MI heart samples; J.P.M.-B. co-supervised experiments and provided technical input into lineage tracing; O.W. carried out the bone marrow and Vavl + cell transplantations; P.R.R. established the project, analysed data and wrote the manuscript.

\section{Additional information}

Supplementary Information accompanies this paper at http://www.nature.com/ naturecommunications

Competing financial interests: The authors declare no competing financial interests

Reprints and permission information is available online at http://www.nature.com/ reprintsandpermissions/

How to cite this article: Balmer, G. M. et al. Dynamic haematopoietic cell contribution to the developing and adult epicardium. Nat. Commun. 5:4054 doi: 10.1038/ ncomms5054 (2014).

(1) This work is licensed under a Creative Commons AttributionNonCommercial-NoDerivs 3.0 Unported License. The images or other third party material in this article are included in the article's Creative Commons license, unless indicated otherwise in the credit line; if the material is not included under the Creative Commons license, users will need to obtain permission from the license holder to reproduce the material. To view a copy of this license, visit http://creativecommons. org/licenses/by-nc-nd/3.0/ 